\title{
MicroRNA-Expression Profiling in Myopia: A Meta-Analysis and Systematic Review
}

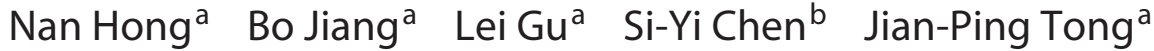 \\ aThe Department of Ophthalmology, First Affiliated Hospital, School of Medicine, Zhejiang University, Hangzhou, \\ PR China; ${ }^{b}$ School of Medicine, Deakin University, Geelong, VIC, Australia
}

\section{Keywords}

microRNA · Myopia $\cdot$ Profiling $\cdot$ Meta-analysis

\begin{abstract}
Background: Myopia (nearsightedness) is currently the most common human eye disorder, worldwide. In the recent years, several studies have addressed the role of microRNAs (miRNAs) in the pathogenesis of myopia. Objectives: The aim of this study was to perform a meta-analysis on the miRNA-expression profiling studies in myopia to identify commonly dysregulated miRNAs in myopic tissues. Method: Seven independent studies were included in the meta-analysis. A vote-counting strategy was employed as the meta-analysis method. Kyoto Encyclopedia of Genes and Genomes (KEGG) pathway enrichment analysis and Gene Ontology (GO) functional enrichment analysis were performed to identify the pathways most strongly affected by the dysregulated mouse miRNAs. Results: According to the vote-counting method, eighteen miRNAs were reported in at least 2 studies with the consistent direction, of which 13 miRNAs were commonly upregulated in myopic samples compared with control samples, and five miRNAs were commonly downregulated. Subgroup analyses divided and compared the differentially expressed miRNAs according to species
\end{abstract}

karger@karger.com www.karger.com/ore

Karger"
(C) 2021 The Author(s)

Published by S. Karger AG, Basel

This is an Open Access article licensed under the Creative Common Attribution-NonCommercial-4.0 International License (CC BY-NC) (http://www.karger.com/Services/OpenAccessLicense), applicable to the online version of the article only. Usage and distribution for commercial purposes requires written permission. (human and animal) and ocular tissue types. The KEGG analysis showed that the dysregulated mouse miRNAs were most enriched in extracellular matrix-receptor interaction signal pathway. The most enriched GO process regulated by the dysregulated mouse miRNAs was cellular protein modification process. Conclusions: Our meta-analysis recommends several miRNAs may provide some clues of the potential biomarkers in myopia. Further mechanistic studies are warranted to elucidate the biological role of the dysregulated miRNAs in the development of myopia.

(c) 2021 The Author(s).

Published by S. Karger AG, Basel

\section{Introduction}

Myopia (nearsightedness) is currently the most common human eye disorder worldwide; it involves a refractive error of the eye and results in blurred vision. High myopia (myopia with a refractive error less than $-6.00 \mathrm{D}$ ) exaggerates the risk of sight-threatening complications including open-angle glaucoma, retinal detachment, chorioretinal atrophy, as well as choroidal neovascularization, which could lead to irreversible visual loss and seriously impact the quality of life of individuals during their most productive years $[1,2]$. The prevalence of myopia
Correspondence to:

Jian-Ping Tong, idrtong@zju.edu.cn 
has reached more than $80 \%$ of young adults in certain East Asian regions. The number of myopic people is estimated to reach 4.8 billion by 2050 , worldwide [3]. The exact pathogenesis of myopia remains unclear. In recent years, microRNAs (miRNAs) have been speculated to be involved in the development of myopia [4].

MiRNAs are endogenous, noncoding, single-stranded regulatory RNA molecules of 17-25 nucleotides which processed from 70 to 100 nucleotide hairpin pre-miRNAs [5]. It is currently deemed that miRNAs play an important regulatory role in multiple cellular biological processes such as cell proliferation, apoptosis, metabolism, and angiogenesis [6,7]. Mature miRNAs can bind to the $3^{\prime}$ untranslated region of one or more target mRNAs that are then cleaved, thereby suppressing protein translation. A single miRNA can have hundreds of target mRNAs; conversely, one mRNA can be targeted by multiple miRNAs [8]. MiRNAs have been found in various cellular tissues and stably present within microvesicles (exosomes) in bodily fluids (e.g., the plasma, urine, vitreous humor, aqueous humor, and saliva) [9-11]. Altered miRNA expression was reported in various complex human diseases and has been studied as therapeutic targets or disease markers aiding clinical diagnoses [12-14].

Over the last decade, miRNA-expression profiling studies on myopia have been performed in animal models and human aqueous humor samples for investigating target candidates and putative pathogenesis of myopia [1521]. These studies identified different amounts of differentially expressed (either upregulated or downregulated) miRNAs between control and myopic ocular samples. However, there is an inconsistency between results because of different profiling platforms applied, different normalization or significance thresholds employed by studies, and tissue samples diversity. As a result, to determine which miRNAs are potential biomarkers of myopia remains a challenge. Therefore, meta-analysis of all available bioinformatic information is essential for identification of the most relevant miRNAs. A vote-counting strategy has been successfully applied in several meta-analyses in searching for potential mRNA or miRNA biomarkers previously, which gave us insights into the meta-analysis of myopia-related miRNA-expression profiling studies [22-25].

In the present study, we performed a meta-analysis of the published microarray studies of myopia. This study aimed to determine the miRNA meta-signature of myopia and to assess the physiological impact of miRNA dysregulation in myopia pathogenesis by pathway enrichment analysis.

MiRNA Profiling in Myopia

\section{Methods}

\section{Search Strategy}

Several electronic literature databases (Pubmed and Web of Science) were used to find and collect miRNA-expression profiling studies on myopia published from inception to June 2020. MeSH terms and keywords were used, using the following search terms: myopia, myopic, miRNA, microRNA, and miR. Only studies published in English were included. Potentially relevant publications were manually retrieved by reviewing abstracts and titles. All references cited in the full text were examined carefully by researchers to find more eligible studies.

\section{Selection Criteria}

Eligible studies had to meet the following inclusion criteria: (1) studies must have the miRNA-expression profiling on humans or animal models of myopia, and report the relative miRNA expression via miRNA microarray, next-generation sequencing or qRT-PCR; (2) studies have to compare intraocular tissue samples obtained from myopia animal models or humans with corresponding control tissues; (3) studies have to report cutoff criteria of differentially expressed miRNAs, sample sizes, and fold changes. MiRNA profiling studies using cell lines, case reports, review articles, or non-English language articles were excluded.

\section{Data Abstraction}

The following eligibility items from the full text and corresponding online supplementary data (for all online suppl. material, see www.karger.com/doi/10.1159/000521300) of each included study were gathered and recorded: author, year of publication, animal model type, tissue types, sample sizes, the platform of miRNA expression profiling, cutoff criteria of dysregulated miRNAs, the list of upregulated and downregulated miRNAs, and corresponding fold change (if available). All miRNA names from various studies were standardized by miRBase version 21 .

\section{Quality Assessment}

We carried out a systematic review and meta-analysis according to the Preferred Reporting Items for Systematic Reviews and Meta-Analyses (PRISMA) statement (online suppl. Table S1) [26]. The quality of each included study was independently assessed according to a quality assessment item list (online suppl. Table S2). Two independent researchers (N.H. and G.L.) evaluated the data and resolved their disagreements by the principal investigator (J.P.T.). For each item, the study was classified as "yes" or "no" which meant "low risk" or "high risk" of bias. Thus, studies with a "yes" score $<5$ were classified as low quality, and studies with a "yes" score $\geq 6$ were classified as high quality. Studies with high quality were included in this metaanalysis.

\section{Meta-Analysis}

Vote-Counting Ranking

A list of differentially expressed miRNAs was produced of each included study comparing miRNA expression between myopia and control samples. The standard vote-counting strategy for ranking potential molecular biomarkers developed by Griffith et al. [22] and Chan et al. [23] was adopted in the meta-analysis. Dys- 
Fig. 1. Flow diagram of the study selection process.

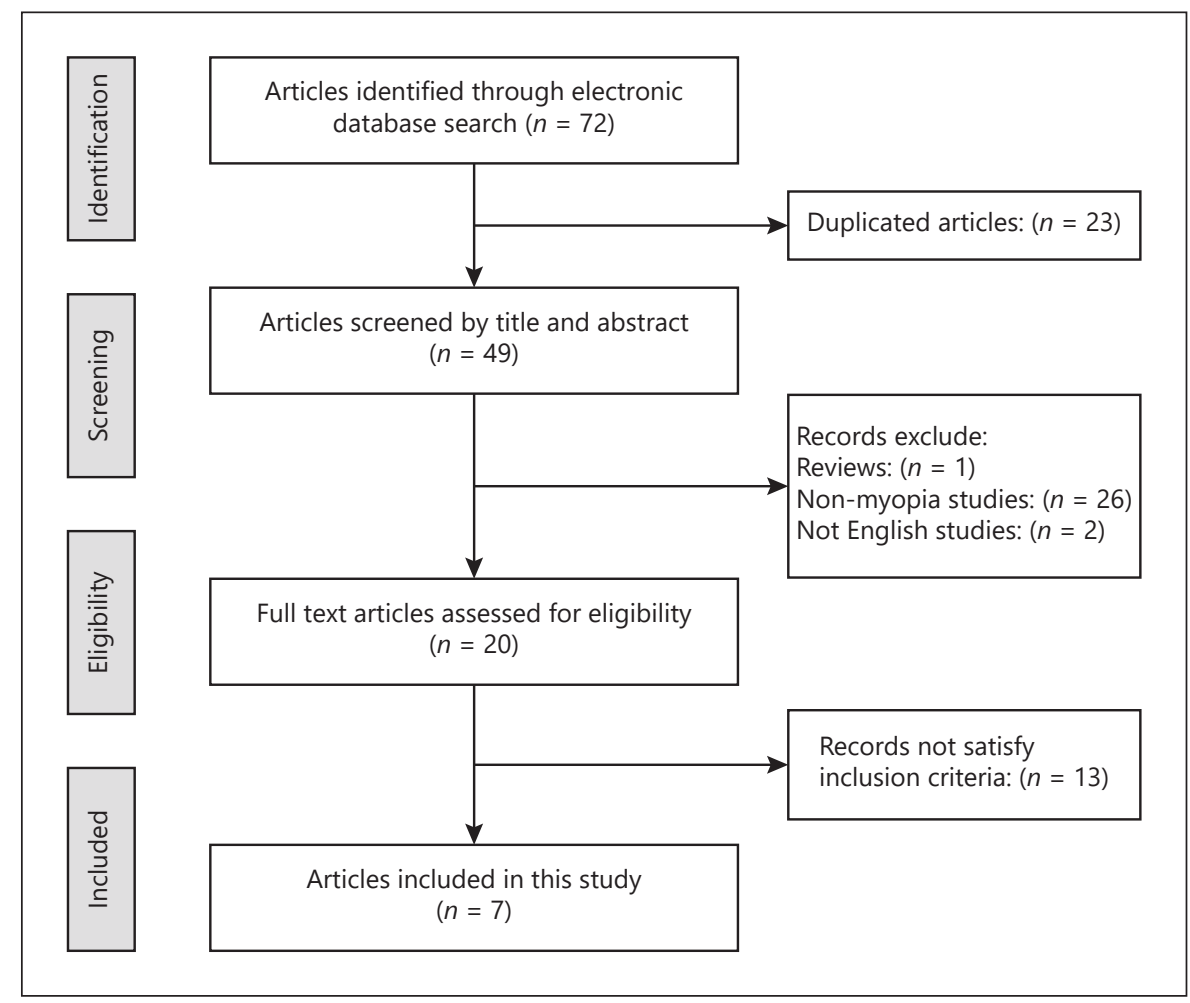

regulated miRNAs in each study were ranked according to the following order of importance: (1) number of studies reported the differentially expressed miRNA with a consistent direction of change; (2) total number of samples; and (3) average fold change (studies with available fold change information). All the ranking and comparisons were performed by the Statistical Product and Service Solutions (SPSS 19.0 for windows; SPSS, Inc., Chicago, IL, USA).

\section{Subgroup Analysis}

MiRNAs express differentially among species, diseases, and tissue types, with different properties and heterogeneities. Subgroup analyses divided and compared the differentially expressed miRNAs according to species (human and animal), tissue types (cornea, retina, sclera, iris, lens, retina, RPE, aqueous humor).

\section{Bioinformatic Analysis}

To access regulatory roles and identify molecular pathways controlled by the ten dysregulated miRNAs in the myopic mouse model, online open software DIANA-miRPath v3.0 (www.microrna.gr/miRPathv3) was used [27]. Functional analysis of commonly dysregulated miRNAs was done for Kyoto Encyclopedia of Genes and Genomes (KEGG) pathways and Gene Ontology (GO) biological process terms [27]. For the enrichment analysis method, unbiased empirical distributions with a microT threshold of 0.7 and false discovery rate correction were used. Molecular pathways were considered significantly enriched when $p<0.05$. Heatmaps of significantly enriched pathways were constructed using the DIANA-miRPath v3.0 tool [27].

\section{Results}

\section{Study Selection and Characteristics of the Included Studies}

The initial search identified a total of 72 articles related to the topic, among which 23 duplicated articles were removed. The residual 49 publications were subsequently intensively assessed by reading titles, abstracts, and full texts, and 42 records that did not satisfy inclusion criteria were excluded. Thus, we eventually included 7 publications in the meta-analysis (Fig. 1). Details of the selected studies features are shown in Table 1.

\section{MiRNA Meta-Signature of Myopia}

In the seven selected miRNA-expression profiling studies, 60 myopic samples and 56 control samples were analyzed. Since the complete list of dysregulated miRNAs of Zhu et al. [21] is not available (Accession "GSE142359" is currently private and is scheduled to be released on December 15,2021$)$, the total number of differentially expressed miRNAs in the seven studies could not be analyzed. According to the vote-counting method, among 44 differentially expressed miRNAs that were reported in at least two studies, 18 miRNAs had a consistent direction (13 were upregulated (Table 2) and 5 downregulated (Ta- 


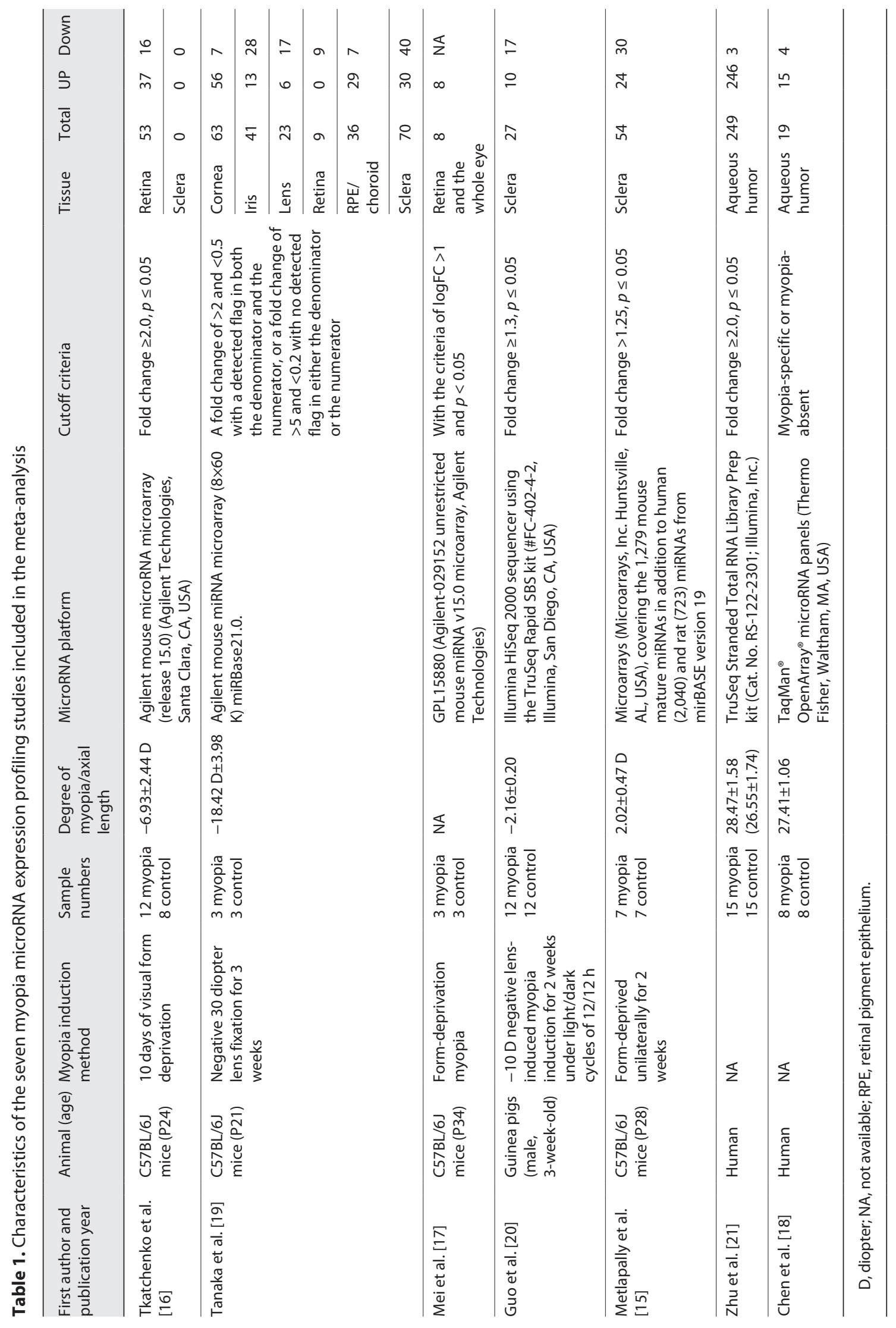


Table 2. Consistently upregulated miRNAs reported in at least two expression profiling studies

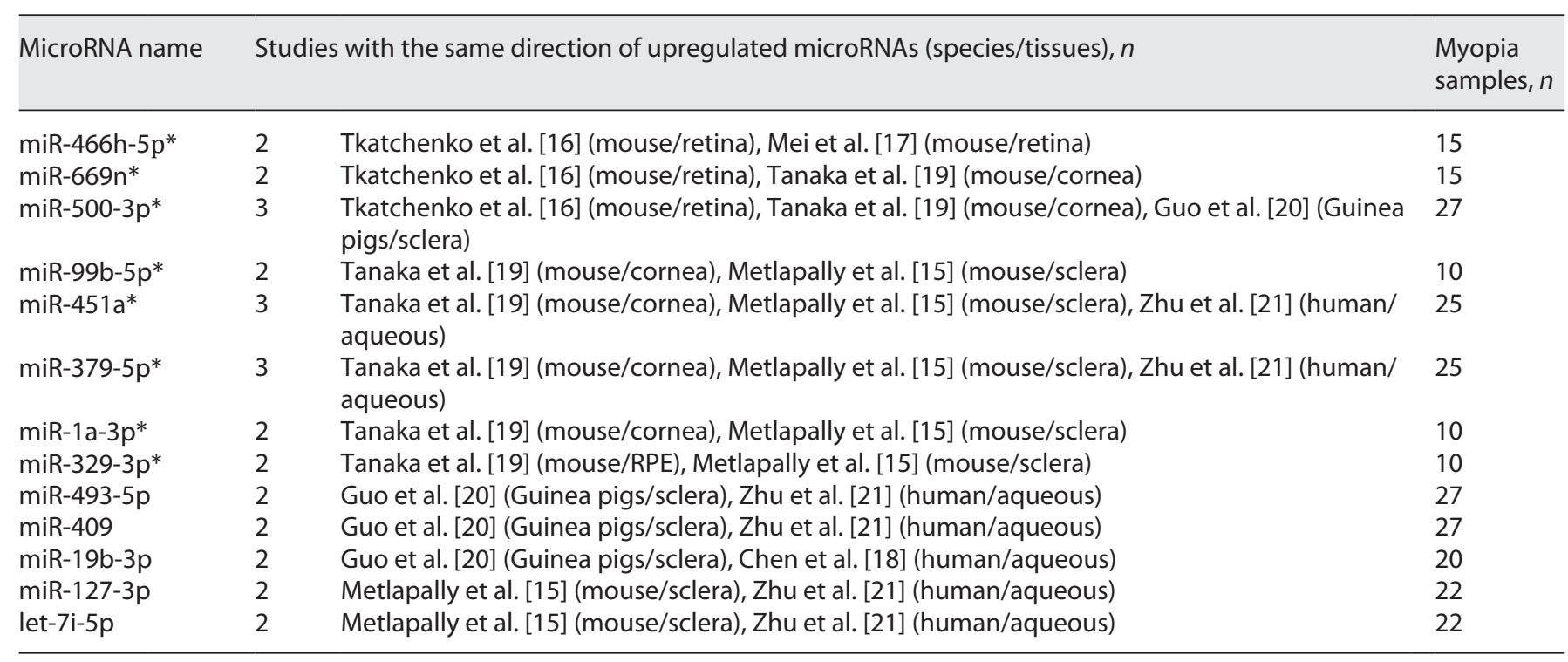

* Consistently upregulated mouse miRNAs reported in at least two expression profiling studies.

Table 3. Consistently downregulated miRNAs reported in at least two expression profiling studies

\begin{tabular}{lllr}
\hline MicroRNA name & \multicolumn{2}{c}{ Studies with same direction of downregulated miRNAs (species/tissue), $n$} & $\begin{array}{l}\text { Myopia } \\
\text { samples, } n\end{array}$ \\
\hline miR-671-5p* & 2 & Tkatchenko et al. [16] (mouse/retina), Tanaka et al. [19] (mouse/retina) & 15 \\
miR-145-5p & 2 & Tkatchenko et al. [16] (mouse/retina), Zhu et al. [21] (human/aqueous) & 27 \\
miR-598-3p* & 2 & Tanaka et al. [19] (mouse/iris, RPE, sclera), Metlapally et al. [15] (mouse/sclera) 10 \\
miR-511-3p & 2 & Tanaka et al. [19] (mouse/sclera), Guo et al. [20] (Guinea pigs/sclera) & 15 \\
miR-143-3p & 2 & Tkatchenko et al. [16] (mouse/retina), Zhu et al. [21] (human/aqueous) & 27 \\
\hline
\end{tabular}

*Consistently downregulated mouse miRNAs reported in at least two expression profiling studies.

ble 3)). The 26 inconsistently reported miRNAs are listed in online supplementary Table S3. Variation in these studies is due to differences in the species investigated, the tissue examined platforms, the number of samples, and statistical analysis approaches used.

\section{Subgroup Analyses}

Subgroup Analysis of Species

Of the seven miRNA-expression profiling studies, two investigated human miRNAs, five investigated animal (4 mice and 1 guinea pigs) miRNAs. In the 4 mice miRNAexpression profiling studies, 255 differentially expressed miRNAs were reported in 25 myopic mouse samples and 21 control mouse samples, among which 157 were reported to be upregulated and 98 downregulated. According to the vote-counting method, 21 differentially expressed miRNAs were reported in at least two studies. In the 21 differentially expressed miRNAs, 10 miRNAs had a consistent direction, of which 8 were reported to be upregulated (Tables 2) and 2 downregulated (Table 3). The 11 inconsistently reported miRNAs are listed in online supplementary Table S4.

Since the complete list of dysregulated miRNAs of Zhu et al. [21] is not available (Accession "GSE142359" is currently private and is scheduled to be released on December 15,2021), the number of differentially expressed miRNAs could not be analyzed. Among the two human miRNA-expression profiling studies, no same differentially expressed miRNA was reported. 
Fig. 2. Heatmap of KEGG pathways enriched in the ten dysregulated mouse myopia miRNAs created by DIANA-miRPath v3.0. The intensity of color represents the FDR-corrected $p$ value. microT-CDS (v5.0) was used for target prediction, $p$ value threshold 0.05, microT threshold 0.7. FDR, false discovery rate.

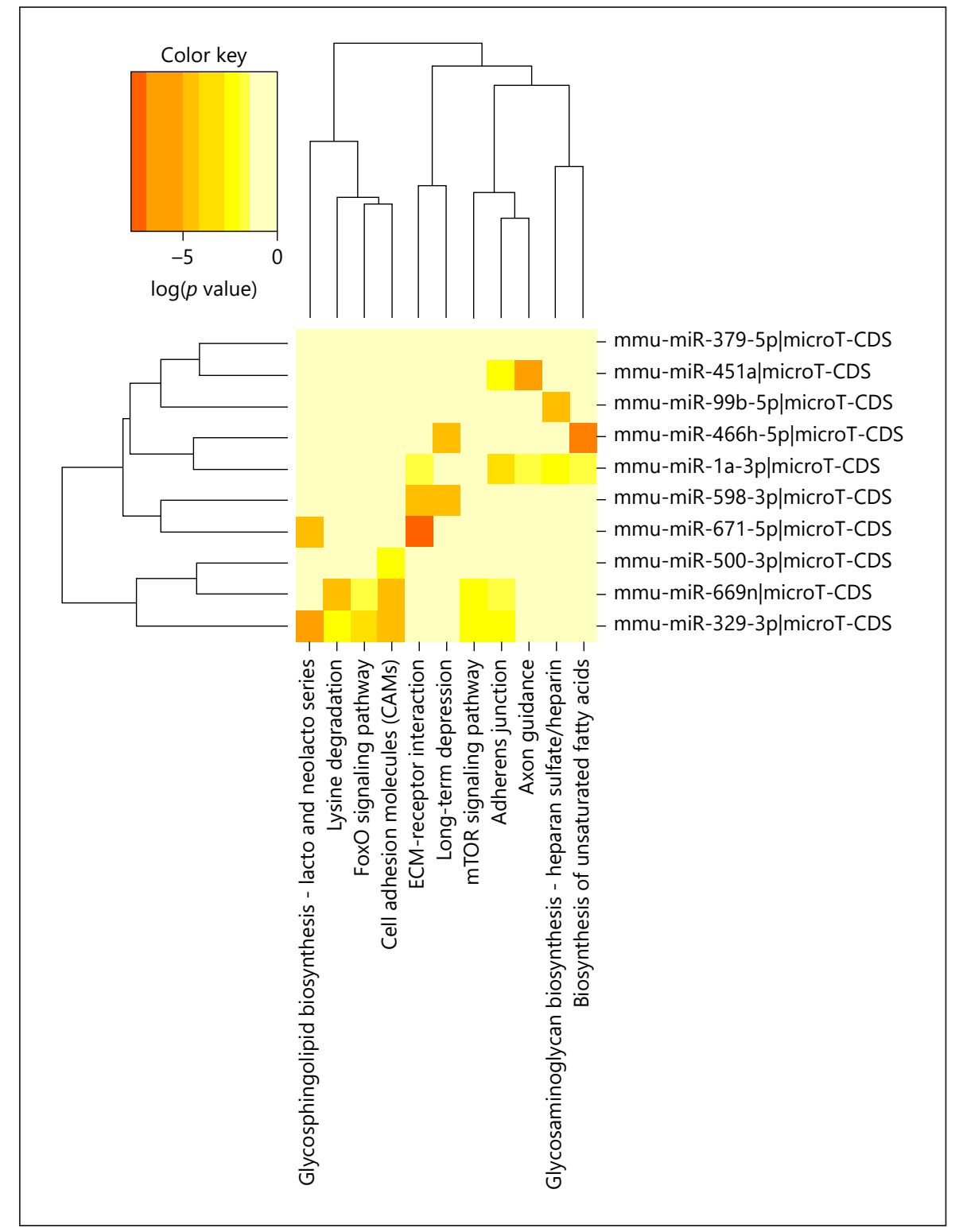

\section{Subgroup Analysis of Ocular Tissues}

Of the seven miRNA-expression profiling studies, three investigated retinal tissue miRNA, four investigated scleral tissue miRNAs, one investigated lens tissue miR$\mathrm{NA}$, one investigated iris tissue miRNAs, one investigated RPE tissue miRNA, one investigated cornea tissue miRNAs, and two investigated aqueous humor miRNAs. Four miRNAs were reported to have a consistent direction in the same tissue. MiR-466h-5p was upregulated in the mouse retina, miR-671-5p was downregulated in the mouse retina, miR-598-3p was downregulated in the mouse sclera, miR-511-3p was downregulated in the sclera of mouse and guinea pigs.

\section{Functional Analysis and Pathway Annotation}

Enrichment analysis of the KEGG pathway and GO function for the ten differentially expressed mouse miRNAs were performed with a significance threshold of $<0.05$. Eleven KEGG pathways were significantly enriched in the myopia miRNA meta-signature, extracellular matrix (ECM)-receptor interaction (mmu04512) was the most enriched signaling pathway (Table 4). The heat maps of most overlapping enriched KEGG pathways in the myopia miRNA meta-signature are (shown in Fig. 2). The most enriched GO processes regulated by the ten dysregulated mouse miRNAs include cellular protein modification process (GO:0006464), cell morphogenesis 
Table 4. List of KEGG pathways enriched in mouse myopia miRNA meta-signature

\begin{tabular}{|c|c|c|c|c|}
\hline No. & KEGG pathway & $p$ value & Genes & miRNAs \\
\hline 1 & ECM-receptor interaction (mmu04512) & $1.039029 \mathrm{e}-07$ & 7 & 3 (mmu-miR-1a-3p, mmu-miR-671-5p, mmu-miR-598-3p) \\
\hline 2 & Adherens junction (mmu04520) & $3.376343 e-05$ & 27 & $\begin{array}{l}4 \text { (mmu-miR-669n, mmu-miR-451a, mmu-miR-1a-3p, } \\
\text { mmu-miR-329-3p) }\end{array}$ \\
\hline 3 & Cell adhesion molecules (CAMs) (mmu04514) & $9.489443 e-05$ & 33 & 3 (mmu-miR-669n, mmu-miR-500-3p, mmu-miR-329-3p) \\
\hline 4 & Long-term depression (mmu04730) & 0.000256563 & 6 & 2 (mmu-miR-466h-5p, mmu-miR-598-3p) \\
\hline 5 & $\begin{array}{l}\text { Glycosphingolipid biosynthesis - lacto and neolacto series } \\
\text { (mmu00601) }\end{array}$ & 0.0008419401 & 5 & 2 (mmu-miR-329-3p, mmu-miR-671-5p) \\
\hline 6 & Axon guidance (mmu04360) & 0.002890375 & 14 & 2 (mmu-miR-451a, mmu-miR-1a-3p) \\
\hline 7 & Biosynthesis of unsaturated fatty acids (mmu01040) & 0.01003442 & 5 & 2 (mmu-miR-466h-5p, mmu-miR-1a-3p) \\
\hline 8 & mTOR signaling pathway (mmu04150) & 0.01136567 & 19 & 2 (mmu-miR-669n, mmu-miR-329-3p) \\
\hline 9 & Lysine degradation (mmu00310) & 0.02140307 & 11 & 2 (mmu-miR-669n, mmu-miR-329-3p) \\
\hline 10 & $\begin{array}{l}\text { Glycosaminoglycan biosynthesis - heparan sulfate/heparin } \\
\text { (mmu00534) }\end{array}$ & 0.02417985 & 3 & 2 (mmu-miR-99b-5p, mmu-miR-1a-3p) \\
\hline 11 & FoxO signaling pathway (mmu04068) & 0.03634205 & 30 & 2 (mmu-miR-669n, mmu-miR-329-3p) \\
\hline
\end{tabular}

Table 5. Top 10 enriched gene ontology biological process terms identified by functional analysis of the miRNAs in the meta-analysis

\begin{tabular}{|c|c|c|c|c|}
\hline No. & GO category & $p$ value & Genes & miRNAs \\
\hline 1 & Cellular protein modification process (GO:0006464) & $<1 \mathrm{e}-325$ & 422 & 5 \\
\hline 2 & Cell morphogenesis (GO:0000902) & $<1 \mathrm{e}-325$ & 195 & 6 \\
\hline 3 & Cellular component (GO:0005575) & $<1 \mathrm{e}-325$ & 3,472 & 6 \\
\hline 4 & Anatomical structure formation involved in morphogenesis (GO:0048646) & $<1 \mathrm{e}-325$ & 220 & 6 \\
\hline 5 & Molecular function (GO:0003674) & $<1 \mathrm{e}-325$ & 3,841 & 7 \\
\hline 6 & Cell differentiation (GO:0030154 & $<1 \mathrm{e}-325$ & 720 & 7 \\
\hline 7 & Anatomical structure development (GO:0048856) & $<1 \mathrm{e}-325$ & 990 & 7 \\
\hline 8 & Biological process (GO:0008150) & $<1 \mathrm{e}-325$ & 3,959 & 8 \\
\hline 9 & Ion binding (GO:0043167) & $<1 \mathrm{e}-325$ & 1,338 & 8 \\
\hline 10 & Organelle (GO:0043226) & $<1 \mathrm{e}-325$ & 1,992 & 8 \\
\hline
\end{tabular}

(GO:0000902), and cellular component (GO:0005575). The top ten enriched GO biological processes identified by functional analysis of the miRNAs in the meta-analysis are summarized in Table 5.

\section{Discussion}

With the advancement of high-throughput technologies, a vast amount of information about the complex role of small regulatory molecules (e.g., mRNAs or miRNAs) in the pathogenesis of varied human diseases has been identified in recent years, including myopia. Results of different miRNA-expression profiling studies on myopia are inconsistent, mainly due to differences in expression profiling platforms, small sample sizes, tissue samples, and diversity of data analysis methods. Therefore, the need to integrate results from different studies through critical and systematic analysis to identify the most relevant miRNAs is essential. In the present study, we performed the first meta-analysis of miRNA profiling studies on myopia, to integrate the results from several independent studies, trying to achieve greater statistical power and estimate the variability between the studies. We employed the vote-counting strategy to identify myopiaspecific miRNAs, which could combine differentially expressed gene lists from separate studies. The present study identified 18 differentially expressed miRNAs that have been consistently reported in at least two studies. The targets of these miRNAs may indicate some clues to the roles of miRNAs in the development and pathogenesis of myopia.

Our results revealed that eighteen miRNAs were reported in at least two studies with the consistent direc- 
tion, of which 13 miRNAs are upregulated in myopia compared with control samples, whereas five miRNAs are commonly downregulated. Three miRNAs (miR500-3p, miR-451a, and miR-379-5p) were consistently upregulated in two studies. In Subgroup Analysis of species, eight miRNAs (miR-466h-5p, miR-669n, miR500-3p, miR-99b-5p, miR-451a, miR-379-5p, miR-1a$3 p$, and miR-329-3p) are commonly upregulated in myopia compared with control samples in mouse, whereas two miRNAs (miR-671-5p and miR-598-3p) are commonly downregulated. In subgroup analysis of tissue, miR-466h-5p was upregulated in the mouse retina, miR-671-5p was downregulated in the mouse retina, miR-598-3p was downregulated in the mouse sclera, miR-511-3p was downregulated in the sclera of mouse and guinea pigs. Further real-time polymerase chain reaction studies are warranted to determine the exact relation of these miRNAs to the myopia process. These miRNAs may open a new insight into myopia pathogenesis and biology.

Among the miRNAs we examined, miR-466h-5p showed consistent upregulation in mouse retina in two studies. MiR-466h is a member of the miR-297-669 cluster located in intron 10 of the Sfmbt2 gene [28]. One study showed that miR-466h robustly upregulated $24 \mathrm{~h}$ following middle cerebral artery occlusion surgery [29]. Whereafter, miR-466h-5p was found to play a role in apoptosis induction and modulate the apoptotic pathway by targeting several anti-apoptotic genes in mammalian cells [30, 31]. Apoptosis is an important biological process of photoreceptor cell degeneration in pathologic myopia [32]. The miR-297-669 cluster also includes miR-669n, which was found to show consistent upregulation in two articles in the present meta-analysis, but in different tissues. The exact impact of the miR-297-669 cluster in regulating the pathophysiological process of myopia needs further research.

MiR-671-5p was found to be consistently downregulated in mouse retina tissue in two studies. MiR-671-5p has been shown to downregulate in several tumor tissues such as osteosarcoma tissue, esophageal squamous cell carcinoma tissue and breast cancer, acting as a tumor suppressor to prevent cell growth, invasion, and metastasis [33-35]. High expression of miR-671-5p specifically reduced the expression of oncogene and suppressed tumor progression. In the present study, miR-598-3p showed consistently downregulated in mouse sclera tissue in two studies. Expression profiling studies have reported miR-598-3p downregulation in breast cancer and cystic fibrosis $[36,37]$. The biological role of miR-671-5p and miR-598-3p in ocular tissue has not been studied until now.

Although animal studies may be informative about myopia indicators in humans, animals still differ from humans and miRNAs can be expressed differently between species. In the present study, some miRNAs are upregulated in human samples but downregulated in animal samples, such as miR-543, miR-411-5p, miR132-3p, mir-376c-3p, and miR-155-5p. These results show that solely using animal models is inadequate to identify biomarkers of myopia in humans, although it is informative and has a synergy effect in common miRNAs.

About $50 \%$ of miRNAs in mouse and human samples are expressed in a tissue- and cell-specific manner [38]. In the ocular tissue subgroup analysis, some miRNAs were consistently dysregulated in different ocular tissue, such as miR-99b-5p, miR-451a, and miR-379-5p, which were commonly upregulated in mouse cornea and sclera tissues. However, some other miRNAs were differentially expressed in different types of ocular tissue, such as miR-669o-5p, miR-467c-5p, and miR-669e-5p were upregulated in mouse retina tissue and downregulated in mouse sclera tissue. These results indicate that miRNAs can be differentially expressed in the different ocular tissues or various time points of disease progression of myopia.

It is well established that miRNAs contribute to biological pathway dysfunction by modulating the expression of their target genes in many human diseases, including myopia. As shown in Figure 2, enriched pathway analysis of the genes targeted by the 10 differentially expressed mouse miRNAs in myopia identified several significantly enriched pathways. Among these, the most enriched pathway was ECM-receptor interaction (mmu04512). The ECM comprises collagens, glycoproteins, and proteoglycans, playing a pivotal role in cell extension and lamellae formation. ECM-receptor interaction forms an important part of cell recognition of ECM, modulating downstream signaling cascades to control cell adhesion and cytoskeletal organization [39]. The inappropriate extension of the ocular axis and concomitant declines in scleral strength and thickness caused by ECM remodeling results in the development of myopia. Previous animal studies have revealed collagen type I synthesis would be downregulated in sclera tissue during myopia development of myopic animals [40, 41]. In addition, enriched pathway analysis of the collocated mRNAs of the differentially expressed long noncoding RNAs in the ocular posterior poles of 2 guinea pig myopia models was also enriched in the pathway of 
ECM-receptor interaction, which is consistent with our findings [42].

Our study also suffers from some limitations. First, the relatively small sample size of the included studies might limit the strength of the conclusions of our metaanalysis. Second, the result of this meta-analysis might also be affected by the difference of species and the biological tissue of the included original miRNAs profiling studies; future studies that are based on homogeneous ocular tissue of the same species may provide more solid evidence. Third, we utilized the vote-counting strategy in the present meta-analysis to explore candidate molecular biomarkers by identifying the consistencies in miRNA expression from all the findings. Although robust rank aggregation analysis has been considered as the ideal method of meta-analyzing the miRNA, it is unrealistic to accomplish the method in our study due to the unavailability of raw datasets and the low concordance of interplatform results. Therefore, the vote-counting strategy might be the best option for the present meta-analysis. However, as the first meta-analysis of the available microarray studies on myopia, this study may provide insight for further research and understanding of the pathophysiology of myopia.

To sum up, the present meta-analysis identified thirteen consistently reported upregulated miRNAs and five consistently reported downregulated miRNAs in the myopic group compared with the control group. These specific miRNAs may provide some clues of the potential biomarkers in myopia. However, since most of these miRNAs are studied in animal model, further validation and quantitative mechanistic studies are urgently needed to understand the association of these miRNAs with the molecular pathogenesis of myopia in human beings.

\section{Acknowledgements}

The authors did not receive any financial support to conduct this study.

\section{Statement of Ethics}

All analyses were based on published studies, and thus, no ethical approval and informed consent are required. Nonetheless, the study adhered fully to the Declaration of Helsinki.

\section{Conflict of Interest Statement}

No competing financial interests exist.

\section{Funding Sources}

This study was supported by the grant from the Project of Natural Science Foundation of Zhejiang Province (LY19H120007).

\section{Author Contributions}

Conception and design: Nan Hong, Bo Jiang, Jian-Ping Tong. Collection and assembly of data: Nan Hong, Lei Gu. Data analysis and interpretation: Nan Hong, Lei Gu, Si-Yi Chen. Manuscript writing: Nan Hong, Si-Yi Chen, Jian-Ping Tong. Final approval of manuscript: Nan Hong, Bo Jiang, Lei Gu, Si-Yi Chen, Jian-Ping Tong.

\section{Data Availability Statement}

All data generated or analyzed during this study are included in this article and its online supplementary material. Further inquiries can be directed to the corresponding author.

\section{References}

1 Hong N, Huang BS, Tong JP. Primary silicone oil tamponade and internal limiting membrane peeling for retinal detachment due to macular hole in highly myopic eyes with chorioretinal atrophy. BMC Ophthalmol. 2015 Nov 11;15:165.

2 Ohno-Matsui K, Lai TY, Lai CC, Cheung CM. Updates of pathologic myopia. Prog Retin Eye Res. 2016 May;52:156-87.

3 Holden BA, Fricke TR, Wilson DA, Jong M, Naidoo KS, Sankaridurg P, et al. Global prevalence of myopia and high myopia and temporal trends from 2000 through 2050. Ophthalmology. 2016 May;123(5):1036-42.
4 Jiang B, Huo Y, Gu Y, Wang J. The role of microRNAs in myopia. Graefes Arch Clin Exp Ophthalmol. 2017 Jan;255(1):7-13.

5 Saliminejad K, Khorram Khorshid HR, Soleymani Fard S, Ghaffari SH. An overview of microRNAs: biology, functions, therapeutics, and analysis methods. J Cell Physiol. 2019 May;234(5):5451-65.

$6 \mathrm{Lu}$ TX, Rothenberg ME. MicroRNA. J Allergy Clin Immunol. 2018 Apr;141(4):1202-7.

7 Tafrihi M, Hasheminasab E. MiRNAs: biology, biogenesis, their web-based tools, and databases. Microrna. 2019;8(1):4-27.

8 Bartel DP. MicroRNAs: target recognition and regulatory functions. Cell. 2009 Jan 23; 136(2):215-33.
9 Ragusa M, Caltabiano R, Russo A, Puzzo L, Avitabile T, Longo A, et al. MicroRNAs in vitreus humor from patients with ocular diseases. Mol Vis. 2013;19:430-40.

10 Drewry MD, Challa P, Kuchtey JG, Navarro I Helwa I, Hu Y, et al. Differentially expressed microRNAs in the aqueous humor of patients with exfoliation glaucoma or primary openangle glaucoma. Hum Mol Genet. 2018 Apr 1; 27(7):1263-75.

11 Mori MA, Ludwig RG, Garcia-Martin R, Brandão BB, Kahn CR. Extracellular miRNAs: from biomarkers to mediators of physiology and disease. Cell Metab. 2019 Oct 1; 30(4):656-73. 
12 Shin VY, Chu KM. MiRNA as potential biomarkers and therapeutic targets for gastric cancer. World J Gastroenterol. 2014 Aug 14; 20(30):10432-9.

13 Fridrichova I, Zmetakova I. MicroRNAs contribute to breast cancer invasiveness. Cells. 2019 Oct 31;8(11):1361.

14 Specjalski K, Jassem E. MicroRNAs: potential biomarkers and targets of therapy in allergic diseases? Arch Immunol Ther Exp. 2019 Aug; 67(4):213-23.

15 Metlapally R, Park HN, Chakraborty R, Wang KK, Tan CC, Light JG, et al. Genome-wide scleral micro- and messenger-RNA regulation during myopia development in the mouse. Invest Ophthalmol Vis Sci. 2016 Nov 1;57(14):6089-97.

16 Tkatchenko AV, Luo X, Tkatchenko TV, Vaz C, Tanavde VM, Maurer-Stroh S, et al. Largescale microRNA expression profiling identifies putative retinal miRNA-mRNA signaling pathways underlying form-deprivation myopia in mice. PLoS One. 2016;11(9):e0162541.

17 Mei F, Wang J, Chen Z, Yuan Z. Potentially important microRNAs in form-deprivation myopia revealed by bioinformatics analysis of microRNA profiling. Ophthalmic Res. 2017; 57(3):186-93.

18 Chen CF, Hua K, Woung LC, Lin CH, Chen $\mathrm{CT}, \mathrm{Hsu} \mathrm{CH}$, et al. Expression profiling of exosomal miRNAs derived from the aqueous humor of myopia patients. Tohoku J Exp Med. 2019 Nov;249(3):213-21.

19 Tanaka Y, Kurihara T, Hagiwara Y, Ikeda SI, Mori K, Jiang X, et al. Ocular-componentspecific miRNA expression in a murine model of lens-induced myopia. Int J Mol Sci. 2019 Jul 24;20(15):3629.

20 Guo D, Ding M, Song X, Sun Y, Li G, Li Z, et al. Regulatory roles of differentially expressed microRNAs in metabolic processes in negative lens-induced myopia guinea pigs. BMC Genomics. 2020 Jan 6;21(1):13.

21 Zhu Y, Li W, Zhu D, Zhou J. microRNA profiling in the aqueous humor of highly myopic eyes using next generation sequencing. Exp Eye Res. 2020 Jun;195:108034.
22 Griffith OL, Melck A, Jones SJ, Wiseman SM. Meta-analysis and meta-review of thyroid cancer gene expression profiling studies identifies important diagnostic biomarkers. J Clin Oncol. 2006 Nov 1;24(31):5043-51.

23 Chan SK, Griffith OL, Tai IT, Jones SJ. Metaanalysis of colorectal cancer gene expression profiling studies identifies consistently reported candidate biomarkers. Cancer Epidemiol Biomarkers Prev. 2008 Mar;17(3):54352.

24 Gholaminejad A, Abdul Tehrani H, Gholami Fesharaki M. Identification of candidate microRNA biomarkers in diabetic nephropathy: a meta-analysis of profiling studies. J Nephrol. 2018 Dec;31(6):813-31.

25 Page MJ, McKenzie JE, Bossuyt PM, Boutron I, Hoffmann TC, Mulrow CD, et al. The PRISMA 2020 statement: an updated guideline for reporting systematic reviews. BMJ. 2021;372: n71.

26 Zhou H, Peng C, Huang DS, Liu L, Guan P. microRNA expression profiling based on $\mathrm{mi}$ croarray approach in human diabetic retinopathy: a systematic review and meta-analysis. DNA Cell Biol. 2020 Mar;39(3):441-50.

27 Vlachos IS, Zagganas K, Paraskevopoulou MD, Georgakilas G, Karagkouni D, Vergoulis $\mathrm{T}$, et al. DIANA-miRPath v3.0: deciphering microRNA function with experimental support. Nucleic Acids Res. 2015 Jul 1;43(W1): W460-6.

28 Luo Y, Liang C, Xu Y, Zhang T. MiR-466h-5p induces expression of myocardin with complementary promoter sequences. Biochem Biophys Res Commun. 2019 Jun 18;514(1): 187-93.

29 Druz A, Betenbaugh M, Shiloach J. Glucose depletion activates mmu-miR-466h-5p expression through oxidative stress and inhibition of histone deacetylation. Nucleic Acids Res. 2012 Aug;40(15):7291-302.

30 Druz A, Chu C, Majors B, Santuary R, Betenbaugh M, Shiloach J. A novel microRNA mmu-miR-466h affects apoptosis regulation in mammalian cells. Biotechnol Bioeng. 2011 Jul;108(7):1651-61.

31 Druz A, Son YJ, Betenbaugh M, Shiloach J. Stable inhibition of mmu-miR-466h-5p improves apoptosis resistance and protein production in CHO cells. Metab Eng. 2013 Mar; 16:87-94.
32 Xu GZ, Li WW, Tso MO. Apoptosis in human retinal degenerations. Trans Am Ophthalmol Soc. 1996;94:411-1; discussion 30-1.

33 Li X, Nie C, Tian B, Tan X, Han W, Wang J, et al. miR-671-5p blocks the progression of human esophageal squamous cell carcinoma by suppressing FGFR2. Int J Biol Sci. 2019; 15(9):1892-904.

34 Tan X, Li Z, Ren S, Rezaei K, Pan Q, Goldstein AT, et al. Dynamically decreased miR-671-5p expression is associated with oncogenic transformation and radiochemoresistance in breast cancer. Breast Cancer Res. 2019 Aug 7; 21(1):89.

35 Xin C, Lu S, Li Y, Zhang Y, Tian J, Zhang S, et al. miR-671-5p inhibits tumor proliferation by blocking cell cycle in osteosarcoma. DNA Cell Biol. 2019 Sep;38(9):996-1004.

36 Fu L, Li Z, Zhu J, Wang P, Fan G, Dai Y, et al. Serum expression levels of microRNA382-3p, -598-3p, - 1246 and - 184 in breast cancer patients. Oncol Lett. 2016 Jul;12(1):26974.

37 Ideozu JE, Zhang X, Rangaraj V, McColley S, Levy H. Microarray profiling identifies extracellular circulating miRNAs dysregulated in cystic fibrosis. Sci Rep. 2019 Oct 29;9(1): 15483.

38 Davis BN, Hata A. Regulation of microRNA biogenesis: a miRiad of mechanisms. Cell Commun Signal. 2009 Aug 10;7:18.

39 McCarthy J, Turley EA. Effects of extracellular matrix components on cell locomotion. Crit Rev Oral Biol Med. 1993;4(5):619-37.

40 Gentle A, Liu Y, Martin JE, Conti GL, McBrien NA. Collagen gene expression and the altered accumulation of scleral collagen during the development of high myopia. J Biol Chem. 2003 May 9;278(19):16587-94.

41 Frost MR, Norton TT. Differential protein expression in tree shrew sclera during development of lens-induced myopia and recovery. Mol Vis. 2007 Sep 6;13:1580-8.

42 Geng C, Li Y, Guo F, Wang J, Yue Y, Zhou K, et al. RNA sequencing analysis of long noncoding RNA expression in ocular posterior poles of guinea pig myopia models. Mol Vis. 2020;26:117-34. 